\title{
Probably Related to Intervention
}

National Cancer Institute

\section{Source}

National Cancer Institute. Probably Related to Intervention. NCI Thesaurus. Code C53259.

A characteristic used to qualify the adverse event as likely related to the medical intervention. According to WHO causality assessment criteria of suspected adverse reactions it is applicable to a clinical event, including laboratory test abnormality, with a reasonable time sequence to the medical intervention, unlikely to be attributed to concurrent disease or other medical interventions, and which follows a clinically reasonable response on withdrawal (dechallenge). Rechallenge information is not required to fulfill this definition. 